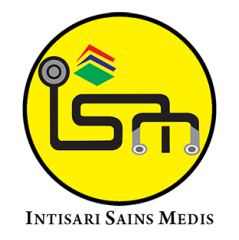

Published by Intisari Sains Medis

\section{Incidence report of traumatic brain injury in Mimika Public Hospital Papua}

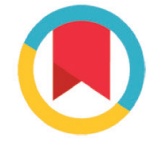

CrossMark

\author{
Kevin Kristian Putra ${ }^{1,4 *}$, Putu Ayu Indra Shanti Wardani ${ }^{2}$, I Wayan Niryana ${ }^{3}$
}

\begin{abstract}
'Internship at Mimika Public Hospital, Mimika, Papua;

${ }^{2}$ Department of Surgery and Anesthesiology, Mimika Public Hospital, Mimika, Papua;

${ }^{3}$ Department of Neurosurgery, Sanglah General

Public Hospital, Denpasar, Bali;

${ }^{4}$ Faculty of Medicine, Universitas Udayana;
\end{abstract}

\author{
*Corresponding author: \\ Kevin Kristian Putra; \\ Internship at Mimika Public Hospital, Mimika, Papua; \\ Faculty of Medicine, Universitas Udayana; \\ kevinkristianputra@gmail.com
}

Received: 2020-11-09

Accepted: 2021-09-20

Published: 2021-10-20

\section{ABSTRACT}

Introduction: Traumatic brain injury is a functional brain disorder caused by an external force, which giving persistent consequences, progressive, long-term care, and rehabilitation might be needed. It is also called a "silent epidemic" due to the increased global incidence rate, socio-economic burden, and quality of life. The major cause of TBI was road traffic injury, fall, and blunt trauma.

Methods: This research was conducted with a descriptive research design using a prospective crosssectional design. The research sampling was using medical records in accordance with inclusion and exclusion criteria from 2018 to 2019.

Keywords: Traumatic brain injury, incidence rate, mechanism of injury, Mimika.

Cite This Article: Putra, K.K., Wardani, P.A.I.S., Niryana, I.W. 2021. Incidence report of traumatic brain injury in Mimika Public Hospital Papua. Intisari Sains Medis 12(3): 724-727. D0I: 10.15562/ism.v12i3.853
Result: The collected sample was 393 cases. There were $66.16 \%$ and $67.18 \%$ cases occurred to man and productive age with incidence rate 178.89 in 100.000 people. The major cause of TBI was road traffic injury and intentional injury with $58.52 \%$ and $24.17 \%$ consecutively. The involvement of alcohol in TBI cases was found in $24.42 \%$ of cases. The usage of the helmet was not found in $96.6 \%$ of cases of RTI.

Conclusion: The majority of TBI patients at Mimika Public Hospital in 2018-2019 occurred in men and in the productive age, which was majorly caused by road traffic injury and intentional injury.

\section{INTRODUCTION}

Trauma is defined as an injury or damage caused by external force and becoming a major cause of morbidities and mortality rates in both developed and developing countries. The usual cases were road traffic accidents (RTA), falls from height, occupational injuries, and assault. ${ }^{1,2}$ According to WHO, RTA was the $8^{\text {th }}$ leading cause of death in all ages and the highest cause of death for children and young adults 5-29 years of age. The death rate was three times higher in low-income countries than in high-income countries. ${ }^{3}$ The greatest contributor to death and disability among all trauma-related injuries is traumatic brain injury (TBI), often noticed as a "silent epidemic" that remains a major public health concern due to the effect on socio-economic and quality of life. When there are changes in cerebral physiology caused by external forces like mechanical, chemical, electrical, or thermal heating to the skull structure, it is called TBI. The effect was persistence, progressive, and long-term rehabilitation might be needed. TBI was classified into three severity levels, which are mild, moderate, and severe, which are based on the level of consciousness measured by the Glasgow Coma Scale (GCS). The severity of TBI can determine the management, intervention, and outcome for the patients. The use of other diagnostic tools, like CT-Scan or ultrasonography, are used to determine other causes or effects that might cause before or occur after trauma. The main purpose of TBI management is to prevent upcoming secondary brain injury. ${ }^{2,4}$

In general, the incidence rate related to brain trauma reported worldwide in the meta-analysis study was 295 per 100.000 people per year. The incidence across Europe with 287.2 per 100.000 hospital admissions/year and the United States with 787.1 per 100.000 emergency department visits/year were higher than the incidence reported in the Middle East Region with 45 per 100.000 hospital 
with limited access of transportation. The health resources are very limited, especially in Mimika Public Hospital, although it is located downtown. Overall, in Mimika, there were no neurosurgeon, orthopedic, or trauma surgeons available. The Radiology facility downtown only had plain x-ray and ultrasonography. CT-Scan was available in late 2019. Mimika is the densest region in Papua province with 95.50 People per $\mathrm{km}^{2}$ with 219.689 people. ${ }^{9}$ In this dense population, the use of private transportations, like cars and motorcycles, has increased rapidly, unlike the availability of public transportation. The use of safety protocols also lacked data. The increased use of transportations and the application of the safety protocol, which is still questionable, were contributing to the increased number of patients with TBI. Despite the increased case of TBI, there was a lack of evidence that might explain the increase of TBI patients. Understanding the key factors of the increased TBI cases might be the keystone to the intervention in management and prevention in public health to reduce the burden of TBI, especially in rural or limited health resources areas. ${ }^{2,5,10}$ This study aims are to give the basic and appropriate data of characteristics and causes of TBI in Mimika.

\section{METHODS}

The data was collected retrospectively from trauma patients between December 2018 and November 2019 at Mimika Public Hospital. Medical record data were collected using the ICD-10 code for
Traumatic Brain Injury (S06) from the patient registration list. Then the hardcopy from the selected medical record number was collected and selected according to the inclusion and exclusion criteria. The analysis of the final selected sample was done with SPSS version 20. The sample size was determined by consecutive method sampling. The inclusion criteria are patients who are diagnosed with TBI with all severity, mild, moderate, and severe TBI. The exclusion criteria are the medical record that cannot fulfill the necessary variables. The variables that were collected are the demographic data like age and gender, mechanism of injury, the severity of TBI, use of the helmet (motorcyclerelated injuries), and alcohol involvement. The collected sample is 393 samples. This study had been reviewed and accepted by the ethical clearance committee of the Medical Faculty of Universitas Udayana with ethical clearance No. 416/ UN14.2.2.VII.14/LP/2020.

\section{RESULTS}

\section{Sample}

Based on the data collected, we found 507 cases that were recorded with the ICD10 code for TBI (S06). After sampling, 393 cases that met all the study criteria were collected for final analysis. From the collected data, there were $66.16 \%$ of males, and 33.84 females with 260 and 133 cases of TBI were reported, respectively. According to the data, we also found various ages, which occur from 6 months old baby to 80 years old elderly. The average age was 25.35 years old (95\%CI: 23.889-
26.812; $\mathrm{p}<0.05)$. The age variable was then divided into three groups, based on the productive age, into not yet productive, productive age, and unproductive age, with respectively 0-17 years, 18-64 years, above 65 years old. The TBI commonly occurred in the productive age followed by the not-yet productive age group with respectively $67.18 \%$ and $31.3 \%$.

\section{Demographic and Incidence Rate}

In 2018-2019, there were 393 reported cases of TBI. Based on sex profile, reported TBI cases were dominantly by men, with $66.16 \%$ cases. Followed by age profile, the productive age group was dominant with $67.18 \%$. The mean age was 25.35 years which is also classified into productive age. Traumatic Brain Injury was classified into three groups of severity: mild TBI, moderate TBI, and severe TBI. The classification was divided based on the Glasgow Coma Scale (GCS) score. Overall, Mild TBI was the highest case of TBI with $91.35 \%$, and only 2 cases of severe TBI were reported. Mimika had 219.689 people. Based on the 393 reported cases in 2018-2019 and the population of Mimika, the incidence rate of TBI in Mimika was 178.89 in 100.000 cases.

\section{Mechanism of Injuries}

The mechanism of injury was divided into three groups, such as road traffic injuries (RTI), Intentional injuries, and Unintentional injuries. Road traffic injuries were single and multiple vehicle accidents, including the pedestrian who might be involved in the accident. Intentional

Table 1. Variable characteristics of TBI in 2018-2019

\begin{tabular}{|c|c|c|c|c|c|c|c|c|}
\hline \multirow{2}{*}{ Variables } & \multicolumn{2}{|c|}{ Mild } & \multicolumn{2}{|c|}{ Moderate } & \multicolumn{2}{|c|}{ Severe } & \multicolumn{2}{|c|}{ Total } \\
\hline & $\Sigma$ & $\%$ & $\Sigma$ & $\%$ & $\Sigma$ & $\%$ & $\Sigma$ & $\%$ \\
\hline \multicolumn{9}{|l|}{ Gender } \\
\hline Male & 227 & 63.2 & 31 & 96.9 & 2 & 100 & 260 & 66.16 \\
\hline Female & 132 & 36.8 & 1 & 3.1 & 0 & 0 & 133 & 33.84 \\
\hline \multicolumn{9}{|l|}{ Age } \\
\hline $0-17$ years & 114 & 31.8 & 8 & 25 & 1 & 50 & 123 & 31.29 \\
\hline 18-64 years & 239 & 66.6 & 24 & 75 & 1 & 50 & 264 & 67.18 \\
\hline$\geq 65$ years & 6 & 1.7 & 0 & 0 & 0 & 0 & 6 & 1.53 \\
\hline \multicolumn{9}{|l|}{ Mechanism of injury } \\
\hline Road traffic injury & 202 & 56.3 & 26 & 81.2 & 2 & 100 & 230 & 58.52 \\
\hline Unintentional injury & 66 & 18.4 & 2 & 6.2 & 0 & 0 & 68 & 17.3 \\
\hline Intentional injury & 91 & 25.3 & 4 & 12.5 & 0 & 0 & 95 & 24.17 \\
\hline
\end{tabular}


Table 2. Mechanism of injury of TBI in Mimika 2018-2019

\begin{tabular}{|c|c|c|c|c|c|c|c|c|}
\hline \multirow{2}{*}{ Variables } & \multicolumn{2}{|c|}{ Road traffic injury } & \multicolumn{2}{|c|}{ Unintentional injury } & \multicolumn{2}{|c|}{ Intentional injury } & \multicolumn{2}{|c|}{ Total } \\
\hline & $\Sigma$ & $\%$ & $\Sigma$ & $\%$ & $\Sigma$ & $\%$ & $\Sigma$ & $\%$ \\
\hline \multicolumn{9}{|l|}{ Gender } \\
\hline Male & 159 & 69.1 & 44 & 64.7 & 57 & 60 & 260 & 66.2 \\
\hline Female & 71 & 30.9 & 24 & 35.3 & 38 & 40 & 133 & 33.8 \\
\hline \multicolumn{9}{|l|}{ Age group } \\
\hline $0-17$ years & 73 & 31.7 & 37 & 54.4 & 13 & 13.7 & 123 & 31.3 \\
\hline 18-64 years & 154 & 67 & 28 & 41.2 & 82 & 86.3 & 264 & 67.2 \\
\hline$\geq 65$ years & 3 & 1.3 & 3 & 4.4 & 0 & 0 & 6 & 1.5 \\
\hline \multicolumn{9}{|l|}{ Alcohol } \\
\hline Yes & 69 & 30 & 3 & 4.4 & 24 & 25.3 & 96 & 24.4 \\
\hline No & 161 & 70 & 65 & 95.6 & 71 & 74.7 & 297 & 75.6 \\
\hline \multicolumn{9}{|l|}{ Helmet } \\
\hline Yes & 8 & 3.5 & - & - & - & - & - & - \\
\hline No & 222 & 96.5 & - & - & - & - & - & - \\
\hline
\end{tabular}

injuries consisted of assault and abuse. Unintentional injuries consist of falls, work-related and sports-related injuries. We found the most common cause of TBI was RTI with $58.52 \%$ cases followed by Intentional injury with $24.17 \%$. TBI caused by RTI and intentional injuries had a higher case with the productive age with $67 \%$ and $86.3 \%$, respectively. However, Unintentional injury commonly occurred in not-yet productive age with $54.4 \%$ of cases. However, the cause of severe TBI was RTI. The use of alcohol and helmet during or before injuries were collected. At least $24.4 \%$ of TBI cases were involved with alcohol usage, and $96.5 \%$ of RTI cases were not using helmets.

\section{DISCUSSIONS}

This study is the first study to describe the incidence of traumatic brain injury in Mimika, Papua. We evaluate and collect the data from a public hospital located downtown of Mimika. Mimika is located in Papua, Indonesia, which has limited access to transportation compared to other developed cities in Indonesia. The health resources were also limited. The experts in certain fields of medicine such as neurosurgeon, orthopedic, radiographer, paramedic, and emergency system, were not yet available or limited. Other diagnostic modalities such as MRI were also not available until the CT Scan in late 2019. Mimika is also the densest population in Papua. The increased population usually paralleled the rapid use of transportation. However, the exact data, safety protocol, and availability of public transportation have a lack of data. This study was to report the incidence rate of TBI in Mimika, evaluate the demographic status, severity, mechanism of injuries, and others that involve the severity of TBI, such as alcohol usage and helmet in RTI cases.

The sample collected was 393 cases that met the inclusion and exclusion criteria. Traumatic brain injury was commonly found in males with $66,16 \%$ cases and productive age with $67.18 \%$ cases. The average age was 25.35 years old. It was supporting the data that TBI mostly occurred in productive age and not-yet productive age groups, with $67.18 \%$ and $31.3 \%$, respectively. In this study, we evaluate the severity of TBI based on the GCS score, which 14-15 is mild TBI, 8-13 is moderate TBI, and below 8 is severe TBI. We found that $91.35 \%$ of cases were Mild TBI, and only 2 cases were reported of TBI. The overall incidence rate in 20182019 was estimated at 178.89 in 100.000 people.

This finding was similar in Lombok and Europe, which stated that the age group of 11-30 years and less than 25 years had the highest case of TBI. Although, in other studies, mean age was found varied, and this variation might occur due to the different analysis as well as inclusion criteria. ${ }^{5}$ The incidence rate was significantly higher than the whole country of Israel but less than Europe with 31.8 in 100.000 people and 260 in 100.000 people respectively. The TBI incidence rate in Mimika cannot be neglected. Because the incidence rate in one rural area in Indonesia had almost six times higher than in Israel, a developed country in middle-east of Asia with more than 8 million people. ${ }^{11,12}$

The cause of TBI had to be followed. The mechanism of injury of TBI was divided into three groups, such as RTI, intentional injury, and unintentional injury. Road traffic injuries were vehiclerelated injuries, either it was single and/ or multiple vehicles that happened in the traffic. Pedestrians that could be involved were also included. For example, such as a single and/or multiple accidents by motorcycle or car or pedestrian that got hit by a vehicle, all the victims that were injured were included and caused by RTI. Intentional injuries such as assault and abuse. Usually, these types of injuries might have alcohol and drug involvement. Although in this study, the use of the drug was not recorded. Unintentional injuries were the type of injuries that happen due to involuntary accidents, such as falls, work, and sport-related injuries. ${ }^{5,11,12}$

From the study, we found the common cause of TBI was RTI followed by intentional injury with $58.52 \%$ and $24.17 \%$, respectively. TBI caused by RTI in productivity has commonly occurred with $67 \%$ cases. However, we found $54.4 \%$ of cases of unintentional injury occurred in the not yet productive age group. The severe TBI was $100 \%$ caused by RTI. The result studies in Lombok, Indonesia, Iran, 
and India all stated that RTI was more common than Falls or physical assault injuries. Meanwhile, $\mathrm{CDC}$ reported that the common cause of TBI were unintentional falls, unintentionally struck by or against objects, and motor vehicle crashes, with $47.9 \%, 17.1 \%$, and $13.2 \%$, respectively. TBI cases caused by falls were most common among the elderly, and RTI was highest among 15-24 years of age. RTI was the $8^{\text {th }}$ leading cause of death for all ages and the leading cause of death for 5-29 years of age. The strong association between risk of RTI death and income level of countries were reported with three times the risk than the high-income countries. Africa and SouthEast Asia have regional rates of RTI death higher than the global rate with 26.6 and 20.7 in 100.000 people, respectively. ${ }^{3,6,10,12}$

The use of safety equipment and alcohol was also recorded because the use of helmets as safety equipment and alcohol might decrease and increase the severity of TBI. We collected the data and found $24.42 \%$ of TBI cases had alcohol involvement. Overwhelmingly, $96.6 \%$ of RTI cases were not using the helmet as part of safety equipment. In recent studies in the pedal cycling community, the use of helmets was associated with a reduction in severe TBI and a lower rate of TBI cases. ${ }^{13}$

This study had several limitations. First, this study of TBI in Mimika, Papua, Indonesia, which was one of many high dense populations in Indonesia with limited health resources, was unable to be described as a full condition of TBI in Indonesia. Indonesia had many regions, different natural challenges, and different healthcare resources. To present characteristics of TBI in Indonesia, different methods and analytical techniques are required to fully represent Indonesia. Second, the data collection of TBI using medical records might not give the detailed characteristics of TBI, such as the onset of injury, how long the patient takes to get into the emergency room, and patient outcome. Third, recollecting the medical record in storage was quite complicated. Each hospital had different storage, possibly different systems in storing the medical record. The medical record for patients whose alive and dead might be stored differently. Furthermore, based on the author's knowledge, this was the first study that describes the characteristic of TBI in Mimika. Hopefully, this study might help other authors to further study the characteristics of TBI in Mimika, Papua.

\section{CONCLUSIONS}

Traumatic brain injury is a "silent epidemic" that remains a major public health concern because of the high burden of socio-economic status for the patient and families. The finding of this study showed that the majority of TBI patients in Mimika Public Hospital in 2018-2019 were men with $66,16 \%$ cases and in the productive age with $67.18 \%$. The major cause of TBI was road traffic injury and intentional injury, with $58.52 \%$ and $24.17 \%$, respectively. The incidence rate of TBI in Mimika was 178.89 in 100.000 people. Alcohol involvement was reported in $24.42 \%$ of cases. The use of helmets was recorded, with $96,6 \%$ of cases of RTI were not using the helmet. Hopefully, these findings may give new insights, whether in clinical and epidemiology of TBI, or in government regulation to reduce the high socio-economic burden of TBI in the community. Further research is necessary to evaluate others and detailed characteristics of TBI.

\section{ETHICAL STATEMENT}

This study had been reviewed and accepted by the ethical clearance committee of the Medical Faculty of Universitas Udayana with ethical clearance No. 416/ UN14.2.2.VII.14/LP/2020.

\section{CONFLICT OF INTEREST}

The authors declare that there is no conflict of interest.

\section{FUNDING}

There was no sponsorship for this research.

\section{AUTHORS CONTRIBUTION}

All authors contributed to the study drafting and design. Preparation, data collection, and analysis were performed by all authors. All authors read and approved the final manuscript.

\section{REFERENCES}

1. Abhilash KPP, Chakraborthy N, Pandian GR, Dhanawade VS, Bhanu TK, Priya K. Profile of trauma patients in the emergency department of a tertiary care hospital in South India. J Fam Med Prim Care. 2016;5(3):558.

2. Syahrul, Imran, Fajri N. Clinical characteristics of traumatic brain injury patients in Dr. Zainoel abidin public hospital Banda Aceh, Indonesia. Bali Med J. 2020;9(1):194-200.

3. World Health Organization. Global Status Report on Road Safety 2018. 2018.

4. Siman-Tov M, Radomislensky I, Knoller N, Bahouth H, Kessel B, Klein Y, et al. Incidence and injury characteristics of traumatic brain injury: Comparison between children, adults and seniors in Israel. Brain Inj. 2016;30(1):83-9.

5. Nguyen R, Fiest KM, McChesney J, Kwon CS, Jette N, Frolkis $\mathrm{AD}$, et al. The international incidence of traumatic brain injury: A systematic review and meta-analysis. Can J Neurol Sci. 2016;43(6):774-85.

6. Tropeano MP, Spaggiari R, Ileyassoff H, Park $\mathrm{KB}$, Kolias AG, Hutchinson PJ. A comparison of publication to TBI burden ratio of low- and middle-income countries versus high-income countries: how can we improve worldwide care of TBI? 2019;47(November):5-10.

7. Kementerian Kesehatan Republik Indonesia. Hasil Riset Kesehatan Dasar 2018. Jakarta; 2018.

8. Badan Pusat Statistik. Papua Province In Figures 2020. Jayapura; 2020.

9. Badan Pusat Statistik. Mimika Regency In Figures. Mimika; 2020.

10. Saatian MR, Ahmadpoor J, Mohamadi Y, Mazloumi E. Epidemiology and Pattern of Traumatic Brain Injury in a Developing Country Regional Trauma Center. Bull Emerg Trauma. 2018;6(1 Jan):45-53.

11. Dewan MC, Rattani A, Gupta S, Baticulon RE, Hung YC, Punchak M, et al. Estimating the global incidence of traumatic brain injury. J Neurosurg. 2019;130(4):1080-97.

12. Rosyidi RM, Priyanto B, Laraswati NKP, Islam AA, Hatta M, Bukhari A, et al. Characteristics and clinical outcome of traumatic brain injury in Lombok, Indonesia. Interdiscip Neurosurg Adv Tech Case Manag. 2019;18(April):100470. Available from: https://doi.org/10.1016/j. inat.2019.04.015

13. Dodds N, Johnson R, Walton B, Bouamra O, Yates D, Lecky FE, et al. Evaluating the impact of cycle helmet use on severe traumatic brain injury and death in a national cohort of over 11000 pedal cyclists: A retrospective study from the NHS England Trauma Audit and Research Network dataset. BMJ Open. 2019;9(9):1-8.

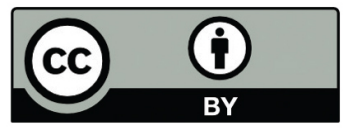

This work is licensed under a Creative Commons Attribution 